\title{
The Resilience of Public Policies in Economic Development
}

\author{
Gonzalo Castañeda ${ }^{1}$ and Omar A. Guerrero $\mathbb{D}^{2,3,4}$ \\ ${ }^{1}$ Centro de Investigación y Docencia Economica (CIDE), Mexico \\ ${ }^{2}$ The Alan Turing Institute, London, UK \\ ${ }^{3}$ Department of Economics and STEaPP, University College London, UK \\ ${ }^{4} \mathrm{CABD}$ N Complexity Centre, University of Oxford, UK \\ Correspondence should be addressed to Omar A. Guerrero; oguerrer@gmail.com
}

Received 3 April 2018; Accepted 2 August 2018; Published 2 October 2018

Academic Editor: Claudio Tessone

Copyright (C) 2018 Gonzalo Castañeda and Omar A. Guerrero. This is an open access article distributed under the Creative Commons Attribution License, which permits unrestricted use, distribution, and reproduction in any medium, provided the original work is properly cited.

\begin{abstract}
This paper studies the resilience of public policies that governments design for catalyzing economic development. This property depends on the extent to which behavioral heuristics and spillover effects allow policymakers to attain their original goals when a particular policy cannot be funded as originally planned. This scenario takes place, for example, when unanticipated events such as natural disasters or political turmoil obstruct the use of resources to advance certain policy issues, e.g., infrastructure or labor reforms. Here, we analyze how the adaptive capacity of the policy-making process generates resilience in the face of disruptions. In order to estimate the allocation of resources across policies, we employ a computational model that accounts for diverse social mechanisms, for example, coevolutionary learning and network interdependencies. In our simulations, we use a data set of 117 countries on 79 development indicators over an 11-year period. Then, we calculate a resilience score corresponding to each development indicator via counter-factual analysis of policy disruptions. Next, we assess whether some development strategies produce resilient/fragile policy profiles. Finally, by studying the relationship between policy resilience and policy priority, we determine which issues are bottlenecks to economic development.
\end{abstract}

\section{Introduction}

Most economists are largely concerned with designing efficient policies for firms and the public sector but much less preoccupied of why certain strategies fail when facing adverse situations. Their focus on the optimal allocation of resources has, on one hand, an ontological explanation since-in the neoclassical view-governments and firms are assumed to have the capacity to control the performance of a system or organization [1]. On the other hand, there is an epistemological explanation since economic theories are deductive constructions built on agents maximizing objective functions that are specified in well-defined problems. These features become evident in the most well-known textbook definition of economics, e.g., "the study of rational behaviors dealing with multiple objectives and limited resources that can be used for alternative purposes" [rephrasing [2]].
Unfortunately, the rational conception of economicswhere consumers, firms, and governments are centrally coordinated through equilibria-does not allow for a clear understanding of why firms, policies, and economies fail. That is, the study of resilience in human-made systems is not possible without a decentralized systemic view. The concept of resilience has different connotations depending on the field of inquiry (e.g., physics, ecology, economics, sociology, or psychology). In this paper, we use a definition that is compatible with the problem of policy-making and development: "Resilience is the [adaptive] capacity of a system, enterprise, or a person to maintain its core purpose and integrity in the face of dramatically changed circumstances" ([3], p. 7).

Under the lens of complexity science, explanations for the failure of economies and their resilience come to light. This is so because complexity builds on the idea that agents within a system and systems themselves are interconnected; 
hence, they are not fully decomposable $[4,5]$. Thus, it is possible for a moderate perturbation on a subpopulation to permeate throughout the system and influence its overall performance with-sometimes-catastrophic consequences. Complexity, however, is not only about connectivity but also about diversity and adaptation. These properties stem from the evolutionary nature of economic systems. With these properties, economies are equipped to respond to adverse events or failures of their components. On one hand, diversity allows a system to generate alternative solutions to the ones impaired by a failure. On the other, adaptation allows agents to react by updating their objectives and strategies when the environment changes.

In this paper, we study the resilience of transformative public policies. A policy is transformative when it is designed to generate a change in a specific issue in order to reach a goal. For example, if the goal is to decrease infant mortality by $50 \%$, a transformative public policy is the construction of hospitals in marginalized communities, while a nontransformative one is the expenditure to maintain the current health infrastructure. When countries attempt to reach a large set of goals simultaneously, resilience arises from the evolutionary nature of the process where policies are designed and implemented. The work of [6] (hereon refer to as CCG) provides a framework to model such process as a behavioral game between a central authority (government) and public functionaries (bureaucrats) on a network of policy spillovers. For a country, the estimated "allocation profile"-i.e., the evolved distribution of resources across policy issues-can be thought of as a consistent (or relatively efficient) package. In this political economy game, the government tries to make the best use of its budget, while bureaucrats may divert some of the allocated funds for a personal gain (i.e., corruption). Hence, rather than modelling agents designing an optimal budgetary allocation, we estimate the emergent allocation profile.

In order to study resilience, we estimate the alternative allocation profiles that emerge in the pretense of adverse events. The intuition is that it is not always feasible to implement the allocation profile estimated for an economic setting that is free of unexpected adversities. These adversities occur when resources initially intended for a transformative policy have to be reassigned to a different purpose. For instance, improving health facilities is put on hold because fighting an epidemic outbreak consumes a large share of resources from the ministry of health; a labor reform has to be postponed when the affected unions sabotage its implementation; an expansion of the highway system is cancelled because recovering from a natural disaster requires substantial resources for repairing damaged infrastructure.

An estimated allocation profile depends on the goals of the government. Consistent with the literature of economic development, we assume that those targets are drawn from exemplary countries. That is, a government determines its goals by imitating the development indicators of a more advanced nation. We term these adopted targets a development mode. Resilience is measured by estimating the allocation profile of a development mode when one of its public policies is exogenously suspended, which we call a disruption.
Therefore, resilience is the capacity of the system to maintain the evolution of socioeconomic indicators in line with a prespecified development mode. For a single country, the allocation profiles corresponding to each development mode allow estimating the expected time of convergence to targets. Significant deviations from the expectation indicate whether a policy is resilient or fragile. In this sense, resilience is not the property of being able to recover the initial condition of a system but rather its capability of continuing to fulfill its goals when facing adverse circumstances.

Resilience analysis allows us to compare not only policy issues but also allocation profiles. For example, a country like Mexico may have different options of development modes (Canada, Singapore, France, etc.); however, some allocation profiles may be more resilient than others. Therefore, assessing the feasibility of a development mode is not only an exercise about costs and benefits but also of endurance to adverse events. This analysis is significantly enriched when considering the priority that governments assign to specific policy issues (i.e., the amount of resources allocated). For instance, a policy issue that is not resilient may hinder the possibility of reaching the targets set by the government. If such a policy receives a low priority from the government, the outcomes in terms of economic development may be worse than expected. In other words, policies with poor resilience and low priority act as bottlenecks to economic development. Identifying these bottlenecks is paramount to development studies. Ironically, due to the rational-equilibrium epistemology of neoclassical economics, there are no adequate quantitative tools for this purpose. Our work fills this gap and provides a well-suited analytic tool.

The rest of the paper is structured with five more sections. In the second section, we frame our approach in the literature of policy resilience. In the third section, we present the data used to calibrate our model. In the fourth section, we provide an overview of the CCG model for analyzing the policy-making process and explain how allocation profiles and their resilience are estimated. In the fifth section, we show the results of our estimations for three country cases and a more detailed description of aggregate outcomes for the entire database. Finally, in the sixth section, we discuss the benefits of complexity tools for studying resilience and summarize our main findings.

\section{On the Nature of Resilience and Policy-Making}

This paper studies the resilience of public policies that are formulated through a process of adaptation and learning. (It is neither about a system's architecture nor of policy tools that can improve the resilience of a particular system (e.g., ecological, environmental, transportation, regional, social, financial, urban, organizational, productive, and business).) It explores the likelihood of failure in the pursuit of specific development modes, independently of how this mode was originally established (e.g., internal political agreements, imitating successful countries, through international consensus, or from pressures of the civil society). This is a relevant and intriguing question since the evaluation of society's (or 
government's) aspirations requires to estimate the reachability of alternative standards. In the related literature, this approach falls under the umbrella of policy resilience at the process level. This literature refers to the institutional and procedural features of policy-making that are in place when an unanticipated shock disrupts a socioeconomic system [7]. In this respect, resilience should be of great concern in setting a societal agenda (development modes) and in formulating an allocation profile that is coherent with such agenda.

The policy process relates to three mechanisms. First, it has to do with the governance of decision-making (e.g., stakeholder participation, institutional accountability, and multilevel and polycentric architectures). Second, it relates to the functionaries' capability of harnessing information for producing workable policies (e.g., involvement of nonstate actors with specialized expertise, a culture of evidence-based decision-making). Third, it deals with the administrative capacity of public officials at the local (or agency) level so that policies can be implemented rapidly and with certain degree of flexibility (e.g., well-designed protocols, use of transparent rules, mechanisms for conflict resolution among agencies, and a well-functioning system for the monitoring and sanctioning of corruption).

The political economy game developed by CCG incorporates important aspects of this policy-making process, emphasizing the distinction between design and implementation. For example, it specifies the mechanisms for the misuse of budgetary allocations by bureaucrats. Accordingly, we employ this model to study how the disruption of a public policy modifies the distribution of resources allocated to the remaining policies, while maintaining the original set of goals. This is a resilience problem because the policy-making process is able to readjust the allocation profile without losing its ability to fulfill the government's agenda (i.e., it is a dynamic property of the system). A related but different concept is robustness. In the literature of complexity, robustness refers to the ability of a system to keep working when some components fail (e.g., an edge in a network). Sometimes, robustness occurs due to the redundancy of components, paths, and functions [8]. Clearly, robustness is a static property of the system since it is not intended to capture the adaptive nature of an economy (it only considers connectivity and diversity).

In recent years, we have witnessed an explosion of quantitative methodologies for analyzing the resilience and robustness of socioeconomic systems $[9,10]$. However, the literature of policy analysis at the process level is mainly qualitative [11-13]. Consequently, the tools and recommendations in this line of research are based solely on case studies and on an informal treatment of complexity. In order to develop a quantitative framework at the process level, it is convenient to construct a calibrated model on how policies are endogenously designed and implemented. This is precisely our approach. First, we build a computational political economy game. Then, we perform Monte Carlo simulations to estimate allocation profiles and resilience scores. Finally, we analyze the outcomes in terms of countries, socioeconomic indicators, development modes, and bottlenecks.

\section{Data}

The data consist of annual observations of 79 policy indicators for 117 countries, covering the 2006-2016 period. Three secondary sources are used to build this database: the Global Competitiveness Report produced by the World Economic Forum, the World Development Indicators, and the World Governance Indicators, being the latter two assembled by the World Bank. Following CCG, we normalize these indicators so that the worst possible outcome takes a value of 0 , while the best value is 1 across countries and years.

We synthesize this information by grouping the 79 development indicators in 13 commonly used pillars. To illustrate the data structure, we pool countries into four clusters by similarity in their development indicators (with cluster 1 being the most advanced and cluster 4 the least). We identify the clusters of countries by applying Ward's method with the L2 (Euclidean) norm as the distance metric across the 79 indicators. Figure 1 shows that, in general, more advanced nations have higher levels of development indicators. The large gap between the clusters 1 and 2 denotes the middleincome development trap.

3.1. Spillover Network. An important component of the CCG model is a network of spillovers between public policies that accounts for well-known interdependencies between policy issues [14-16]. Empirically, this network is estimated from partial correlations between development indicators at the level of each country. Therefore, we estimate one network for each country. Arguably, a network topology reflects the socioeconomic conditions in a specific nation. We employ a two-step empirical strategy developed in the estimation of neural networks from functional magnetic resonance imaging data $[17,18]$. First, we apply the method of triangulated maximally filtered graphs (TMFG) [19] to estimate which pairs of indicators have significant relationships. Then, we determine the edges' directions (inferred causality) through the likelihood-ratios method developed by [20]. The field of network estimation has various different methods, without a broad consensus on a gold standard. Each method assumes a specific underlying model and is designed for data with certain properties. Our methodological choice is based on the applicability of this strategy to high-dimensional time series of short length. However, as new and better methods emerge, we expect further improvements to our estimations.

\section{Methods}

We use the CCG model in order to simulate the policymaking process through a behavioral game with two types of agents: a central authority (government) and public servants (functionaries or bureaucrats). Firstly, the government allocates resources to different public policies, with the aim of improving the indicators associated to their respective policy issues. Secondly, functionaries in charge of implementing these policies have incentives to divert public funds for personal gain. This game takes place on a spillover network. These spillovers encourage free-riding and reinforce a misalignment between the government's and functionaries' 


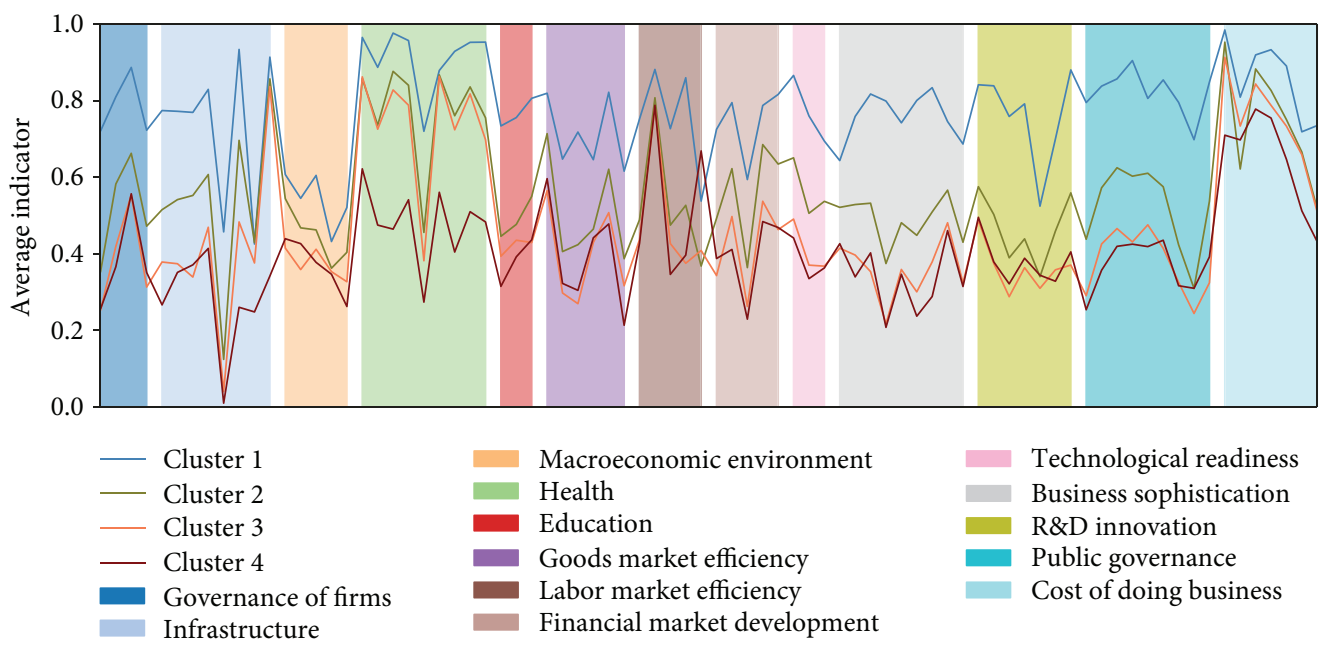

Figure 1: Gaps in socioeconomic indicators between development clusters in 2016. The $X$-axis corresponds to development indicators, organized by development pillar. The wider the shaded area, the more development indicators within the corresponding pillar. For countries without observations for 2016, we take the most recent one.

incentives. (See [6] for a detailed interpretation of the model's equations and different validation tests).

4.1. Evolution of Development Indicators. There are $N$ policy issues in the economy, each one with an indicator that measures its level of development. As a government invests $P_{i}$ $\epsilon[0,1]$ resources in a policy issue, its indicator grows, i.e., the investment accumulates. This means that, when the government sets a target $T_{i}$ for policy issue $i$, indicator $I_{i}$ will reach $\mathrm{T}_{i}$ after a number of periods. Hence, the dynamics describing the evolution of $I_{i}$ are given by

$$
I_{i, t}=I_{i, t-1}+\gamma\left(T_{i}-I_{i, t-1}\right)\left(C_{i, t}+\sum_{j} C_{j, t} A_{j, i}\right),
$$

where $\gamma$ captures the effectiveness of policy-making in a specific country; $C_{i, t} \in\left[0, P_{i}\right]$ is the contribution of bureaucrat $i$ (see explanation in next section), and $\mathbb{A}$ is the adjacency matrix of the spillovers network (a weighted directed graph).

4.2. The Learning Process of Public Servants. In a given period $t$, a public servant $i$ receives $P_{i, t}$ resources from the central authority and uses $C_{i, t}$ effectively in the implementation of the public policy. Hence, $P_{i, t}-C_{i, t}$ is the level of corruption of $i$, while the level of $i$ 's indicator gives him (or her) political status. Then, in order to determine the impact of this contribution, the bureaucrat evaluates the change in benefits $F_{i}$ expressed through

$$
F_{i, t}=\left(I_{i, t}+P_{i, t}-C_{i, t}\right)\left(1-\theta_{i, t} f_{R, t}\right),
$$

where $\theta_{i, t}$ is an indicator function derived from the supervision of the central authority, and $f_{R, t}$ is a map from the indicator of rule of law to a probability. In the CCG model, a period should not be interpreted as time, since the method does not aim at reproducing time series. Instead, it should be interpreted as the realization of events such as budgetary readjustments. Hence, if it takes more periods to reach one set of goals than another, we say that the former are more difficult to attain than the latter.

We assume that the government cannot observe the functionaries' contributions directly, although larger diversions of funds are more difficult to hide. Hence, we model government supervision as a random variable $\theta_{i, t}$. The outcome $\theta_{i, t}$ is 1 if the public servant in policy issue $i$ is caught diverting public funds and zero otherwise. Then, the probability mass function of $\theta_{i}$ at time $t$ is

$$
\theta_{i, t}=\left\{\begin{array}{cc}
1 \quad \text { with probability } f_{C, t} \frac{\left(P_{i, t}-C_{i, t}\right)}{\sum_{j=1}^{N}\left(P_{j, t}-C_{j, t}\right)}, \\
0 \quad \text { with probability } 1-f_{C, t} \frac{\left(P_{i, t}-C_{i, t}\right)}{\sum_{j=1}^{N}\left(P_{j, t}-C_{j, t}\right)},
\end{array}\right.
$$

where $f_{C, t}$ is a function mapping the indicator of control of corruption to a probability.

Note that $f_{C, t}$ captures the efforts from the central authority to detect corrupt officials. Meanwhile, $f_{R, t}$ reflects the capability of the state to punish officials involved in these activities. These two mechanisms describe different constraints that governments face when fighting corruption. To be more specific, $f_{R, t}$ and $f_{C, t}$ take the form

$$
f_{X, t}=\frac{I_{X, t}}{e^{1-I_{X, t}}}
$$

where $X=R$ for the rule of law, or $X=C$ for control of corruption.

The contribution of functionary $i$ is determined by

$C_{i, t}=\min \left\{P_{i, t}, \max \left(0, C_{i, t-1}+d_{i, t}\left|\Delta F_{i, t}\right| \frac{C_{i, t-1}+C_{i, t-2}}{2}\right)\right\}$, 
where $\Delta F_{i, t}$ is the most recent change in benefits, and $d_{i, t}$ is the sign function

$$
d_{i, t}=\operatorname{sgn}\left(\Delta F_{i, t} \cdot \Delta C_{i, t}\right) \text {, }
$$

such that

$$
\begin{aligned}
\Delta F_{i, t} & =F_{i, t-1}-F_{i, t-2}, \\
\Delta C_{i, t} & =C_{i, t-1}-C_{i, t-2} .
\end{aligned}
$$

That is, functionaries' contributions increase when higher (or lower) past benefits coincide with higher (or lower) past contributions. Therefore, as time goes by, bureaucrats learn the level of corruption that gives them certain confidence of keeping the benefits derived from their public post.

4.3. The Adaptive Behavior of the Central Authority. The government's problem consists in deciding how to allocate its limited resources to a large set of policies. Its objective is to reduce the gap between the current indicators $\left(I_{i}\right)$ and their targets $\left(T_{i}\right)$, where the latter come from a previously selected development mode. Formally, the government's multidimensional problem is

$$
\min \left(\sum_{i=1}^{N}\left(I_{i, t}-T_{i}\right)^{2}\right) .
$$

Resource allocations $P_{1, t}, \ldots, P_{N, t}$ are the control variables of the central authority. We call a specific configuration of these variables an allocation profile. These packages of policies are the key endogenous variables to be simulated by the model. The amount of resources that the government can invest per period in a profile is restricted by

$$
\sum_{i}^{N} P_{i, t} \leq B \forall t
$$

where $B$ denotes noncommitted resources of the central authority. It is important to clarify that the resources involved in this problem are those destined to transformative policies, not public expenditure committed to previously established purposes (e.g., highway maintenance, agricultural subventions, and payment of public debt).

Each time step, the central authority determines an allocation profile and evaluates the gap between targets and observed indicators. The amount of resources allocated to policy issue $i$ is determined by

$$
p_{i, t}=\frac{q_{i, t}}{\sum_{j}^{N} q_{i, t}}
$$

where $q_{i, t}$ is the propensity of assigning resources to policy $i$, defined as

$$
q_{i, t}=\left(T_{i}-I_{i, t}\right)\left(K_{i}+1\right)\left(1-\theta_{i, t} f_{R, t}\right),
$$

where $K_{i}$ is the number of outgoing connections of node $i$, also known as its out-degree. Here, the out-degree captures the centrality or importance of a policy issue for the country. Hence, the government does not know the structure of the network but has a proxy of the relevance of each policy issue.

Finally, the amount of resources allocated to policy $i$ is

$$
P_{i, t}=p_{i, t} B
$$

This component of the policy-making process indicates that the government's behavior is adaptive. That is, resources are redistributed when undesired events such as the detection of corruption materialize.

4.4. Algorithm. Once the political economy game is formulated mathematically, an agent-based model is programmed with Algorithm 1. Note that, for each simulation, four endogenous variables are obtained: public servants' contributions and benefits, government's allocations, and society's development indicators. A simulation halts when a convergence criterion is met for all indicators. The combination of a coevolutionary learning process, the diversity of conditions that bureaucrats face, and the network of policy spillovers requires computational simulation.

4.5. Estimation of Allocation Profiles. Each country in the data set can adopt development modes of nations with (1) a higher GDP per capita (adjusted for purchasing power parity) and (2) a higher average level of development indicators. This means that, for the sample of 117 countries, there are more than 6000 combinations of country-mode pairs (e.g., RUS-CAN stands for Russia adopting the Canada development mode). We estimate the allocation profiles of each of these pairs, assuming no policy disruptions. For a given pair, a single allocation profile consists of the intertemporal averages $P=\left\{1 / \ell \sum_{t=0}^{\ell} P_{i, t}\right\}_{i=1}^{N}$, where $\ell$ is the number of periods before converging to the targets. For the same pair, we perform $m$ Monte Carlo simulations and compute the average allocation profile $\bar{P}=\left\{1 / M \sum_{m=1}^{M} P_{i}^{m}\right\}_{i=1}^{N}$. Each simulation is calibrated by imputing the empirical values of the country's development indicators in 2016 (the initial conditions), the estimated network of spillovers, the values of the adopted mode's development indicators in 2016 (the targets), and the budget constrain $B$ obtained from an indicator on public expenditure as a fraction of GDP.

In order to provide inference on aggregate metrics, we calibrate parameter $\gamma$ to fit our endogenous variable of corruption $\left(P_{i}-C_{i}\right)$ to the observed levels of an empirical indicator on the diversion of public funds. This is done through a clustering algorithm that classifies all countries into different categories of $\gamma$ (see details in [6]). Once the model has been calibrated, we obtain the average allocation profile for a set of Monte Carlo simulations. The purpose of estimating these profiles is to obtain the relative priorities that governments give to different policies under 


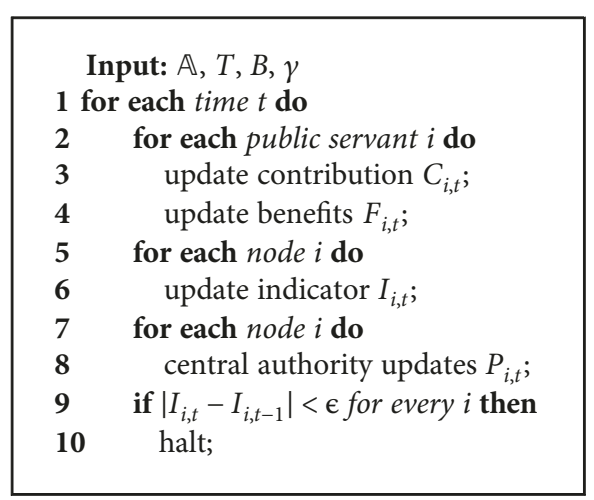

Algorithm 1: Computation implementation.

each development mode, which we use in this paper to identify bottlenecks.

For illustration purposes, Figure 2 presents the estimated policy profiles for three different country-mode pairs. From this example, we infer that policy priorities depend on the specific pair, which highlights the relevance of context in the design and implementation of policies. Likewise, we can conclude that the coherence of the allocation profile, and not the strength of isolated policies, is what really matters to achieve development goals.

4.6. Estimation of Resilience. The strategy to assess the resilience of an individual policy is to measure the effect of disrupting its budgetary allocation. This exercise describes a scenario where the central authority of a country is forced to put off the implementation of a transformative policy due to an unexpected event. In the context of our behavioral game, the disruption of a transformative policy in issue $i$ precludes it from receiving sustained allocations. Aware of this disruption, the government adapts and modifies its policy priorities in an attempt to reach the targets specified by the chosen development mode. In other words, this "change of plans" alters the coevolutionary learning process between central authority and functionaries, and, hence, the dynamics through which the indicators reach all their targets. More formally, assume that policy $i$ has been disrupted $\left(P_{i, t}=0\right.$ for all $t$ ). Let $\mathscr{T}_{j}^{i}$ denote the time it takes for indicator $j$ to converge to its target while $i$ has been disrupted. Then, the adjusted convergence time induced by a disruption of $i$ is

$$
\mathscr{T}^{i}=\frac{\left(T_{i}-I_{i, 0}\right)}{N-1} \sum_{j \neq i}^{N-1} \mathscr{T}_{j}^{i}
$$

where $T_{i}-I_{i, 0}$ takes into account the fact that, by disrupting an indicator with a large initial gap, the average convergence time becomes shorter. The fact that a policy issue is not receiving direct investments does not inhibit the possibility that the level of its associated indicator can increase. On the contrary, this is likely to happen because there are spillover effects among policies.

As we previously discussed, resilience is a dynamic property of a system. This means that the effect of disrupting a particular public policy needs to be measured beyond the budgetary consequences of setting $P_{i, t}=0$. In other words, a policy is said to be resilient if the corresponding adjusted convergence time is lower than the expectation from removing direct investments from $i$. In contrast, the policy is said to be fragile if its adjusted convergence time is higher. (A direct comparison of convergence times between the complete and the disrupted models is not informative. This is so because it is not possible to isolate the effect of the disrupted policy due to the adaptive nature of the model. That is, the complete model has $N$ objectives and the disrupted has $N-1$, rendering any comparison useless without a normalization that accounts for the effect of the disrupted policy.)

Our proxy to measure the direct budgetary effects on convergence time is $\bar{P}_{i}$, the average allocation to policy issue $i$ obtained from the model without disruptions. Note that, by (11), $\bar{P}_{i}$ and $T_{i}-I_{i, 0}$ are positively correlated. Therefore, if the model was purely driven by budgetary effects, a relationship between $\bar{P}_{i}$ and $\mathscr{T}^{i}$ would be almost perfect (although not necessarily linear). Deviations from the expectation of such relationship denote evidence of resilience and fragility. Allow us to illustrate this with a hypothetical example. Figure 3 shows artificial data about the positive relationship between $\mathscr{T}^{i}$ and $\bar{P}_{i}$. The solid line represents the estimated relationship, i.e., the expected budgetary effect on the adjusted convergence time. The dots above the confidence interval denote fragile policy issues because their disruption increase convergence time more than expected. The dots under the interval correspond to resilient policies. Finally, the policy issues inside the interval are undefined because they are explained by the budgetary effect.

Figure 3 exemplifies the importance of a systemic approach to public policy. The points away from the expectation capture the effects derived from the coevolutionary dynamics of the model and the network. Given that each country can choose between multiple development modes and that it has a unique combination of network topology, initial conditions, and institutional factors, the relationship to be estimated for each country is

$$
\widehat{\mathscr{T}}^{i}=a \bar{P}_{i}^{b}+\varepsilon_{i},
$$

where $a$ and $b$ are the parameters to be estimated via nonlinear least squares. Then, we construct our resilience score of policy $i$ as follows

$$
\mathscr{R}^{i}= \begin{cases}\hat{\mathscr{T}}_{l}^{i}-\mathscr{T}^{i} & \text { if } \mathscr{T}^{i}<\widehat{\mathscr{T}}_{l}^{i}, \\ \hat{\mathscr{T}}_{u}^{i}-\mathscr{T}^{i} & \text { if } \mathscr{T}^{i}<\widehat{\mathscr{T}}_{u}^{i}, \\ 0 & \text { otherwise, }\end{cases}
$$

where $\widehat{\mathscr{T}}_{l}^{i}$ and $\widehat{\mathscr{T}}_{u}^{i}$ denote the lower and upper bounds of the estimated confidence interval. For clarity in the exposition of our results, the positive value of this score indicates that the corresponding policy is resilient (below the curve), while the negative value denotes that the policy is fragile (above the curve). 

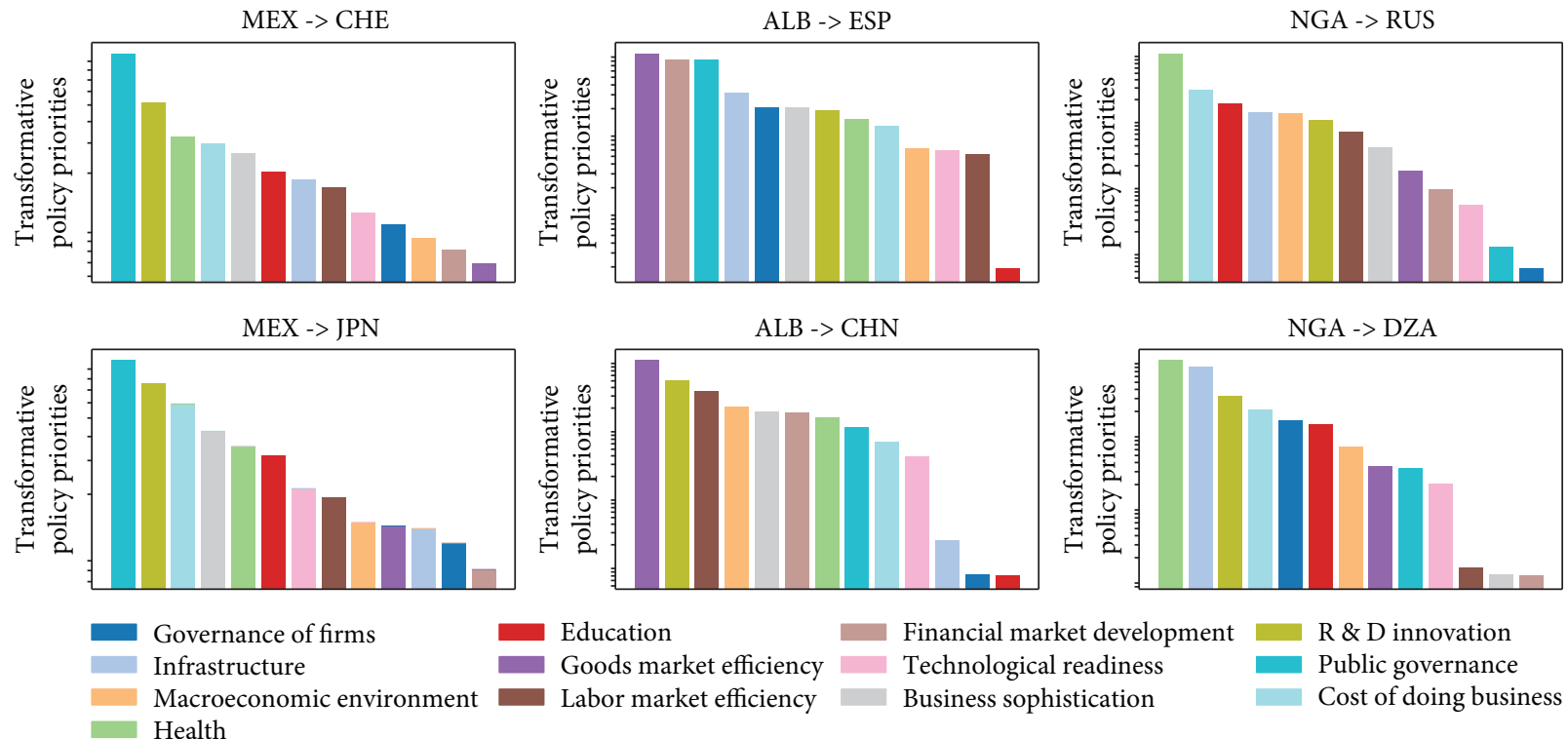

Financial market development Technological readiness Business sophistication

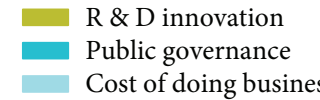

Figure 2: Average allocation profiles of three country cases.

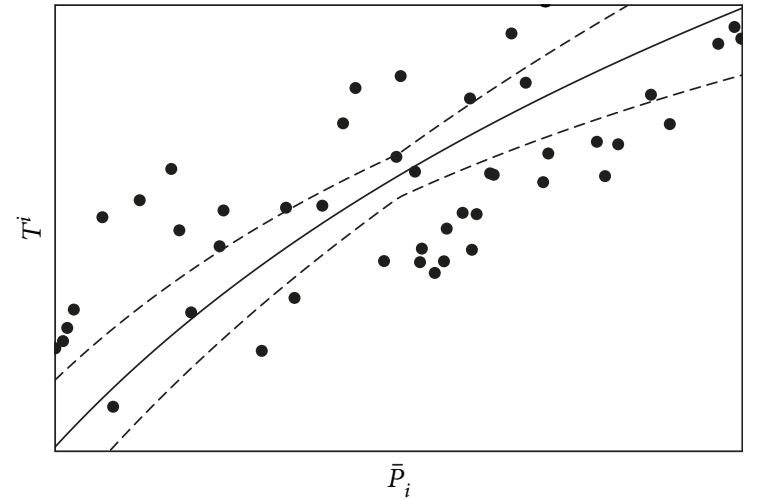

- Hypothetical data

- Estimated relationship

--- Confidence interval

Figure 3: Hypothetical identification of resilient/fragile policies.

\section{Results}

We estimate the 79 disrupted allocation profiles of each country-mode pair (for each of the 79 cases of disruption in a country-mode pair, we performed 30 Monte Carlo simulations). For an individual country $x$, we pool all the outputs from simulating the adoption of all the development modes that $x$ can follow. Then, we fit the curve in (14) to these outputs. Although we do not display confidence intervals in our plots (due to their narrowness), all our results were calculated considering an interval of $95 \%$ confidence. This curve provides the expected adjusted convergence time when $x$ cuts its budget allocation to a disrupted policy with certain level of priority. When the convergence time from disrupting a policy deviates significantly from this expectation, it suggests resilience or fragility.
First, we provide an aggregate example in Figure 4 by fitting (14) to the pooled outputs of all the country-mode pairs. (The time variable in the vertical axis is normalized by the sum of adjusted convergence times of all perturbations in the profile, while the priorities in the horizontal axis are normalized by the country's budget $B$.) This aggregate exercise is useful to show the general structure of convergence times and relative priorities. Note that the simulated points produce a nonlinear fit, so resilient policies are those below the curve, while fragile ones lie above it. Many policies exhibit low relative priorities and short convergence times when disrupted. On the other hand, fewer have large budgetary allocations while, at the same time, produce long convergence times. The inset panel shows the distribution of deviations from the curve. This shows that most of the points are well explained by budgetary effects.

5.1. The Importance of Spillovers. Our next aggregate result highlights the importance of spillover effects on the simulation outcomes. Network effects on node-level dynamic were already identified by [6], who showed that more incoming spillovers induce lower contributions. Then, in the context of resilience, a straightforward demonstration of these effects is through the adjusted convergence time when the allocation on issue $i$ is disrupted. Figure 5 shows this metric aggregated by countries (Figure 5(a)) and by indicators (Figure 5(b)). That is, the dots represent average values across simulation runs and development modes for all indicators and for all countries, respectively. In each panel, the estimates have been obtained from the full model, incorporating spillover effects, and from an incomplete version where we deactivate all interactions among policy issues.

Figure 5(a) shows that the simulations generate significantly different convergence times for most countries when we remove spillovers. Notice that, in most cases, the adjusted convergence time is lower when countries have spillover effects. This indicates that network effects tend to be 


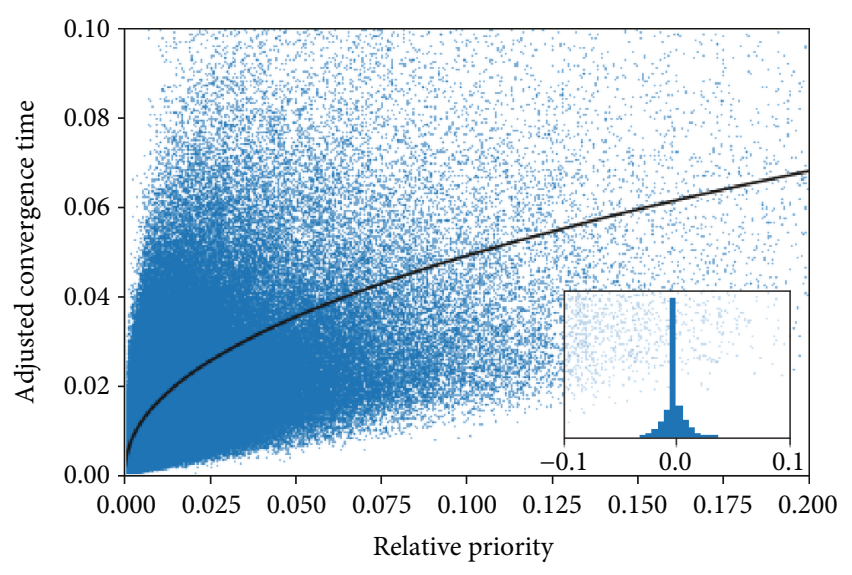

FIGURE 4: Expected convergence time from budgetary effects for all country-modes pairs. The inset panel shows the deviations from the fitted curve.

beneficial for reaching development goals, independently of disrupted public policies. In some cases, however, this does not occur due to different mechanisms. In our view, an intuitive explanation for an increase in convergence time due to spillover effects has to do with the reallocation of resources. For example, it is possible that-for some countries and development modes-redistributing resources from a disrupted issue to other policies generates biases in favor of nodes with relatively fewer outgoing spillovers. Under these circumstances, it may seem as if the presence of spillovers is detrimental for reaching the targets. Interestingly, we find that these cases tend to be advanced nations, suggesting that spillover effects are more beneficial (at least in a more direct way) in developing countries. Of course, second order effects might also contribute to this phenomenon.

In Figure 5(b), we can see that, by removing the spillovers, the adjusted convergence time varies modestly between indicators. This is expected since, in the absence of a network, indicators evolve only through the contributions of their bureaucrats. In this case, most of the empirical variation comes from the different budget constraints across countries. Since indicator-level aggregations include all countries, this variation is not observed across indicators. Therefore, spillovers provide an important source of heterogeneity to study resilience at the aggregate level of each indicator.

\subsection{Simulation Results for Three Countries. Out of the 117} countries in the data set, we select three to estimate (14). These countries are in significantly different stages of development, for example, Mexico is considered a high-middle income country (cluster 2), Albania is lower-middle (cluster 3 ), and Nigeria is low (cluster 4).

Figure 6 shows the pooled outputs of all the countrymode pairs for each of the three cases. To be more precise, each dot in Figure 6(a) corresponds to $\mathscr{T}^{i}$ and $\bar{P}_{i}$ when $i$ is disrupted in a specific development mode that Mexico can adopt. That is, there are 79 dots for each development mode. Here, we highlight the dots corresponding to the most resilient development mode (in blue) and to the least one (in orange). The resilience of a country-mode pair is measured through the average resilience score from its 79 disrupted policies. High resilience at the level of an allocation profile does not imply that all policies are resilient. It means that a country adopting a particular development mode has better chances at reaching its targets below the expected time.

Figure 7 shows the outcome of a similar exercise, this time, highlighting the indicators with the highest and the lowest average resilience scores across development modes. The blue (orange) dots highlight the most (least) resilient indicator across different development modes. In the Mexican case, the most fragile issue falls into the pillar of public governance, while the most resilient belongs to the pillar of cost of doing business. For Albania, the most resilient indicator corresponds to the R\&D innovation pillar and the most fragile to the financial market development pillar. In the case of Nigeria, the most fragile issue relates to the pillar of infrastructure while the most resilient is part of the health pillar. These findings illustrate two important facts: (i) a disaggregated analysis is very important because some policy issues within a development mode can be very resilient while others can be extremely fragile, and (ii) a particular policy issue can be highly resilient (or fragile) for a country, no matter which development mode is adopted.

Finally, Figure $8(\mathrm{a})-8(\mathrm{c})$ show which ones are the most and the least resilient development pillars. The resilience of a pillar measures the average score of its corresponding indicators (or policy issues) across all development modes adoptable by a country. For the Mexican case, public governance is the most fragile pillar while infrastructure is the most resilient. Interestingly, the most resilient (fragile) pillar contains indicators that are rather fragile (resilient), highlighting the importance of disaggregation. This also occurs in the other two cases but with different pillars. For Albania, the most fragile pillar is health, and the most resilient is $R \& D$ innovation. This picture is completely reversed for Nigeria. This is an interesting result since, intuitively, one would expect health to be fragile. The Nigerian case is special because the effect on convergence time is largely dominated by the gap between initial conditions and targets. In fact, Nigeria exhibits a disproportionate indicator-target gap of its health-related indicators, which makes it a high-priority pillar (due to (11)), as shown in Figure 2. In contrast, for example, Mexico's top pillar-public governance-is the most fragile. This highlights the importance of context specificity and the interactions between initial conditions, targets, and spillovers.

5.3. A Global View of Resilience. In this section, we provide a global perspective of resilience by computing average scores at the level of development pillars and indicators. The purpose of this section is to understand how, across all the countries in the sample, certain policy issues tend to be fragile or resilient. These global averages are obtained from all the country-mode estimations. In other words, these results should be interpreted as the level of resilience that we should expect if we were to study a policy issue in a hypothetical country with the average characteristics of the population. 


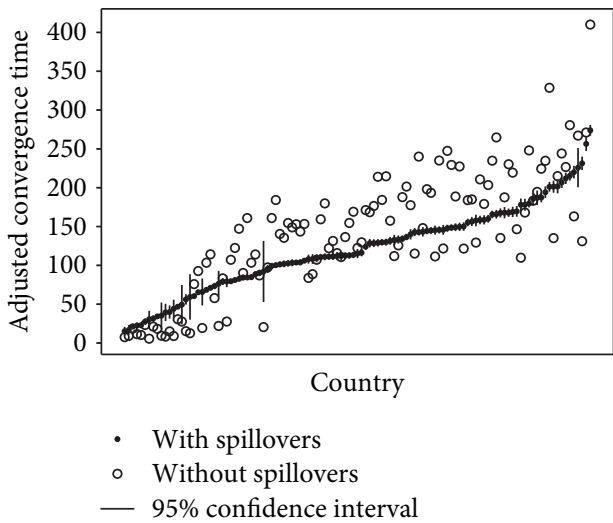

(a)

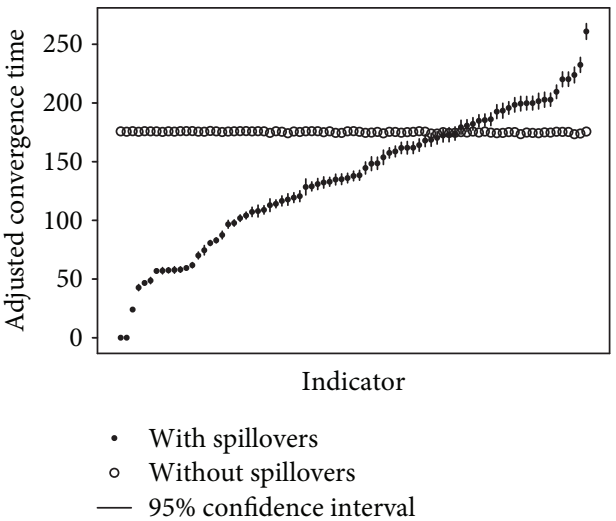

(b)

FIGURE 5: Estimations for the adjusted convergence times in models with and without spillovers. (a) Aggregation by country (sorted by $\mathscr{T}$ ). (b) Aggregation by indicator (sorted by $\mathscr{T}$ ).
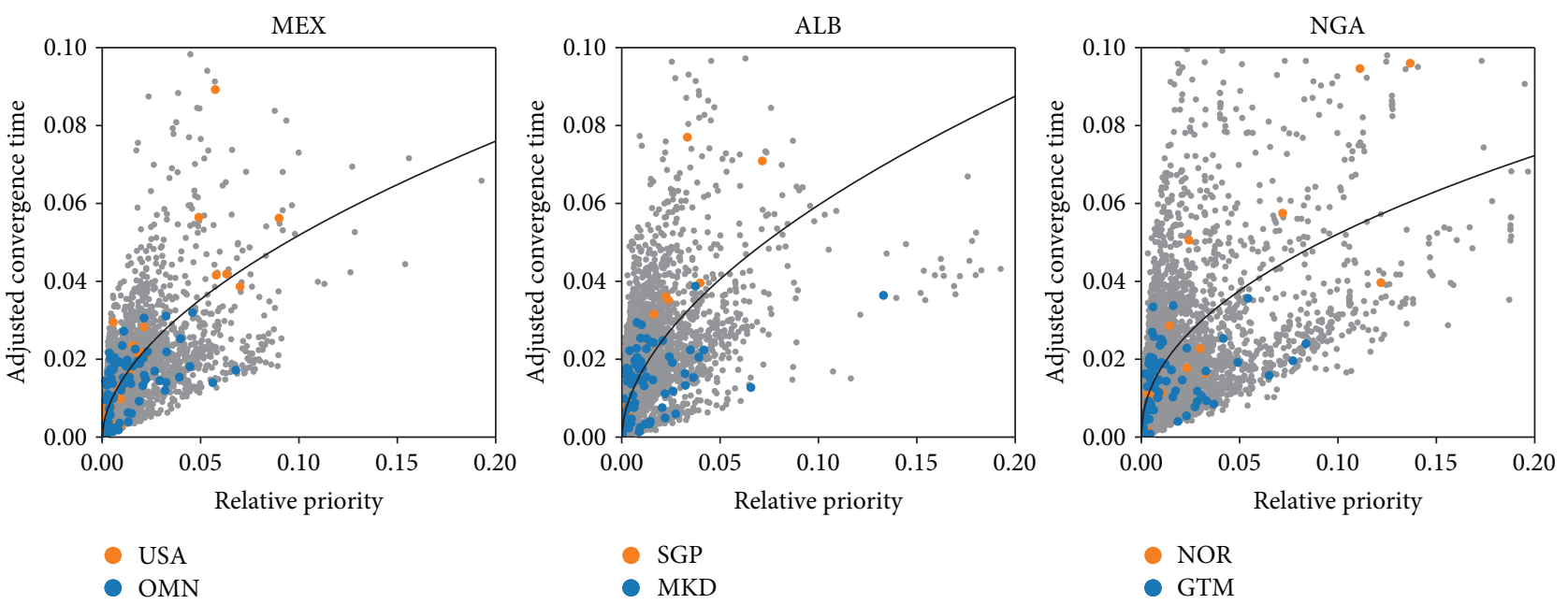

Figure 6: The most (blue) and the least (orange) resilient development modes for three countries. The majority of orange dots are not visible because they are covered by the blue ones. Gray dots refer to other development modes.

Figure 9 shows the average resilience scores at the level of development pillars. The top three most resilient pillars are governance of firms, technological readiness, and cost of doing business. In contrast, the three most fragile pillars (negative resilience scores) are infrastructure, macroeconomic environment, and business sophistication. Note that, due to the dominance of laggard economies in the country population, these estimates are biased towards developing countries. Nevertheless, if we pick a country at random, these are the expected levels of resilience to be found in each development pillar.

Figure 10 presents the average resilience scores at the level of each development indicator. Once more, the bar diagram highlights the importance of a disaggregated analysis. For instance, Figure 9 indicates that the macroeconomic environment pillar is rather fragile, yet in Figure 10, we can see that the inflation indicator is resilient. This is particularly interesting given the generalized notion that controlling inflation is central for economic development. This result, in contrast, suggests that this policy issue is not critical to attain the rest of the development goals. This is coherent with the idea of incorporating more diverse development goals in the international agenda. For example, one of the drivers behind the Sustainable Development Goals has been a critical position towards the narrow scope of the Millennium Development Project, which focused almost exclusively in economic outcomes. In fact, most development pillars have at least one indicator with the opposite classification (resilient/ fragile). The top five most resilient indicators are time to resolve insolvency, time required to enforce a contract, government procurement of advanced tech. products, survival to age 65 (male), and adolescent fertility rate, with two of them belonging to the pillar of cost of doing business and two more to health. The top five most fragile indicators are business cost of crime and violence, infant mortality rate, tuberculosis cases, quality of port infrastructure, and quality of roads, with two of them belonging to the health pillar and two more to infrastructure. 


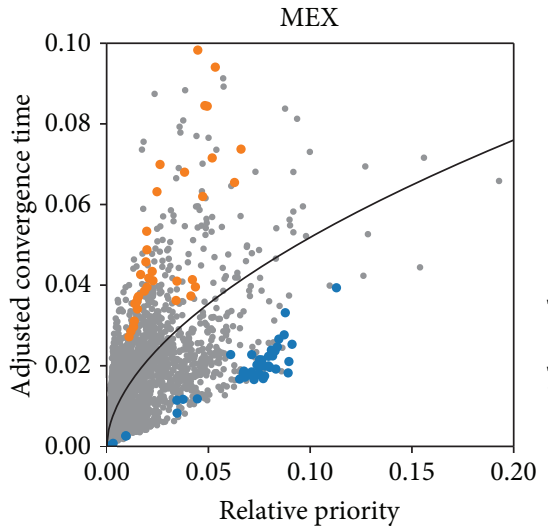

- Property rights

- Business costs of crime and voilence

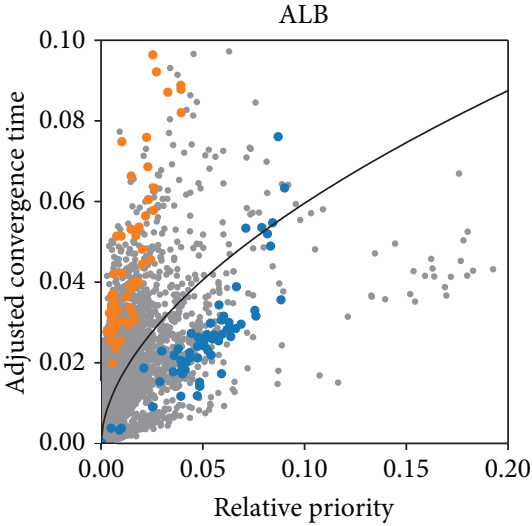

- Venture capital availability

- Quality of scientific research institutions

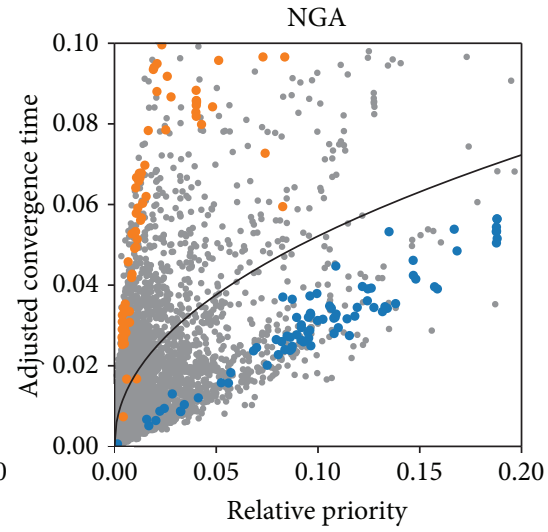

Quality of overall infrastructure

- Infant mortality

FIgURE 7: The most (blue) and the least (orange) resilient development indicators for three countries.
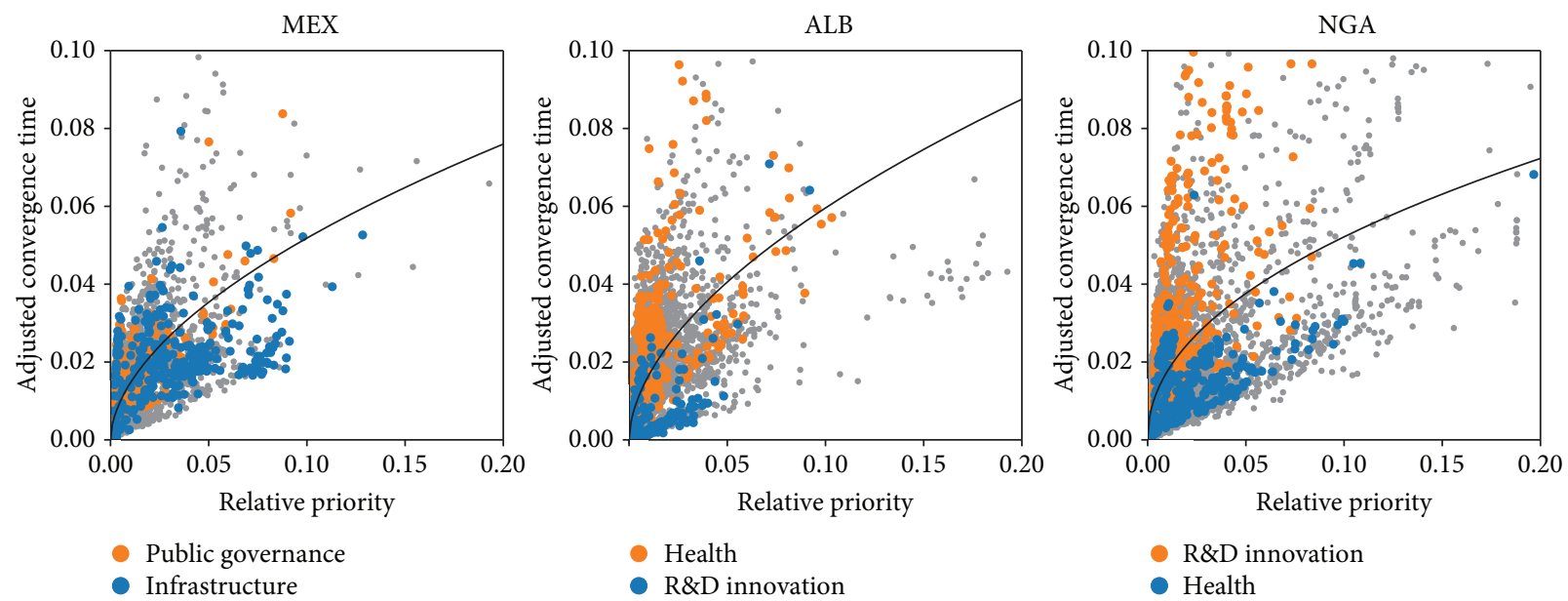

- Public governance

- R\&D innovation

- Health

Figure 8: The most (blue) and the least (orange) resilient development pillars for three countries.

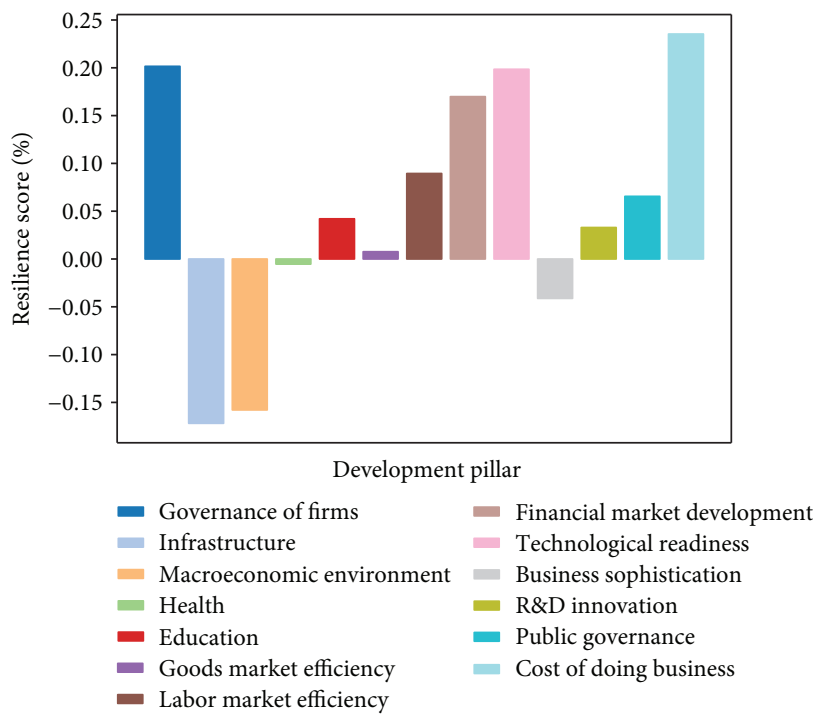

FIGURE 9: Average resilience by development pillar. 


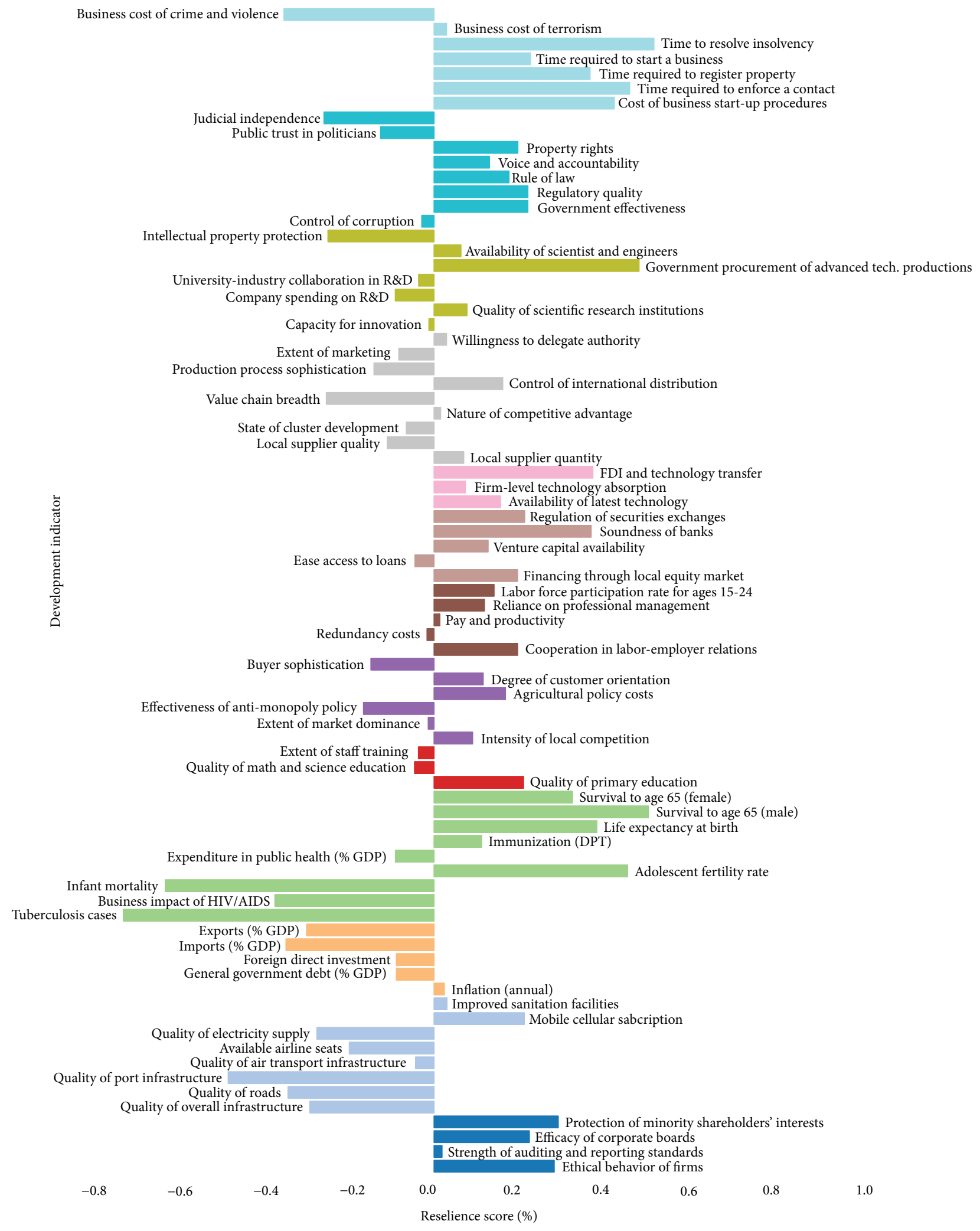

FIgURE 10: Average resilience by development indicator.

5.4. Bottlenecks and Sturdy Policy Issues. One of the key challenges in development is the identification of policy issues that act as binding constraints to development [21-23]. In other words, there exist certain policy issues that act as bottlenecks, so their identification can make policy design substantially more effective. In our context, bottlenecks are 


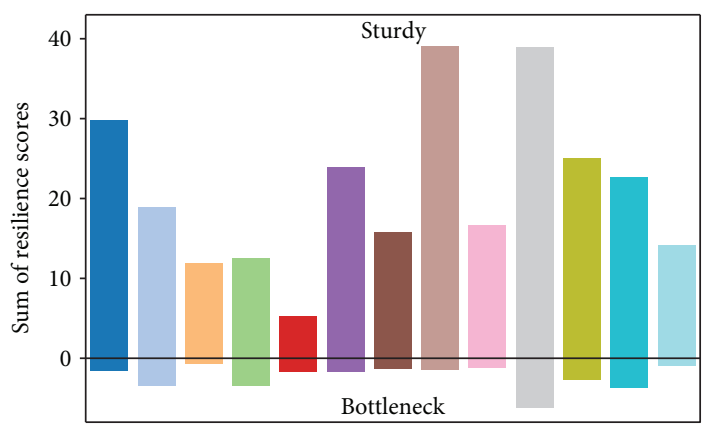

Development indicator

$\begin{array}{ll}\text { Governance of firms } & \text { Financial market development } \\ \text { Infrastructure } & \text { Technological readiness } \\ \text { Macroeconomic environment } & \text { Business sophistication } \\ - \text { Health } & \text { R\&D innovation } \\ - \text { Education } & \text { Public governance } \\ - \text { Goods market efficiency } & \text { Cost of doing business } \\ - \text { Labor market efficiency } & \end{array}$

FIgURE 11: Bottlenecks and sturdy policy overall scores by development pillars. Bottlenecks: fragile policies in the $5^{\text {th }}$ percentile of relative priorities. Sturdy: resilient policies in the $95^{\text {th }}$ percentile of relative priorities.

fragile policy issues that receive a low relative priority by the central authority. Put it differently, these are policies where the central authority does not invest large sums, and, ironically, their disruption lowers the chances of attaining the goals of a chosen development mode.

Another important concept is sturdiness. We say that a policy issue is sturdy when it is a prime objective of the central authority, and, nonetheless, it is very resilient to disruptions. This paradoxical scenario is explained by two important features of the policy-making process. On the one hand, the adaptability of the agents involved in the design and implementation of policies (governments and public functionaries) allows a proper reallocation of resources from the original priorities to alternative policies which, in turn, mitigate negative impacts. On the other hand, the network of interdependent policies helps to improve the performance of an indicator even if it does not receive direct contributions from the general budget.

In order to identify bottlenecks and sturdy policies, we isolate those points that fall in the $5^{\text {th }}$ and $95^{\text {th }}$ percentiles of relative priorities. Bottlenecks are fragile policies in the $5^{\text {th }}$ percentile, while sturdy ones are resilient issues in the $95^{\text {th }}$. Once isolated, we calculate the overall score of the bottlenecks (which is negative) and sturdy policies (positive) at the levels of pillars and development indicators. The overall score consists of summing the indicator-level scores. The reason for a sum, and not an average, is that the sum takes into account the frequency with which a policy appears in bottleneck or sturdy issues across all country-mode pairs. Figure 11 shows the overall scores of bottlenecks and sturdy policies in each development pillar.

The first thing to notice in Figure 11 is the large difference in the number of cases between bottlenecks and sturdy policies, being the latter much more frequent. This pattern emerges from the learning and adaptive behavior of the agents. It means that the adaptive nature of the policymaking process reduces the number of scenarios where adverse events hinder economic development. The second feature is that the sturdiest indicators belong to the pillars of business sophistication, financial market development, and governance of firms. On the other hand, the most critical bottlenecks belong to the pillars of business sophistication, public governance, health, and infrastructure.

Figure 12 presents a similar visualization, disaggregated by development indicators. Here, the sturdiest policy issues are control of international distribution, venture capital availability, financing through local equity market, buyer sophistication, and ethical behavior of firms. In contrast, the most critical bottlenecks are production process sophistication, value chain breadth, extent of staff training, university-industry collaboration, and business impact of HIVIAIDS. The reason why an indicator can be sturdy and a bottleneck at the same time is that resilience scores may be differ significantly between country-mode pairs. Figure 13 in the appendix presents another visualization at the level of the development pillars but disaggregated into countries.

\section{Conclusions}

The quantitative study of prioritization and resilience of public policies demands a systemic approach in order to provide rigorous guidelines for policy advice. Traditional methods such as regression analyses, growth diagnostics, and benchmarking are severely limited for this purpose because they neglect the fundamental systemic qualities of any economy. These include the interdependencies between policies, the importance of societal context (i.e., initial conditions, structure, and development modes to pursue), the multiobjective goal-seeking behavior of governments, and political economy considerations that produce misaligned incentives. In this paper, we develop a complexity approach that overcomes these limitations and use it to understand the phenomenon of resilience in public policy.

Our framework allows identifying the allocation profiles that an adaptive government selects-through a behavioral game-in an attempt to reach the multiple targets of a prespecified development mode. These profiles are established in an environment where public functionaries in charge of implementing policies learn how to divert public funds. Thus, by modelling the policy-making process, our methodology is capable of analyzing the adaptive nature of resilience in policy issues when facing adverse events.

By disturbing the resource allocation to specific policy issues, we obtain multiple results that help us to understand policy resilience. First, some policy issues are resilient (i.e., the development goals are reached earlier than expected) while others are fragile. Second, context matters because the level of resilience or fragility depends on the country's initial conditions and the adopted development mode. Third, for specific countries, some policy issues prove to be resilient across development modes. Fourth, although some development pillars tend to be resilient for specific countries, it is common to find that at least one of its indicators is fragile. 


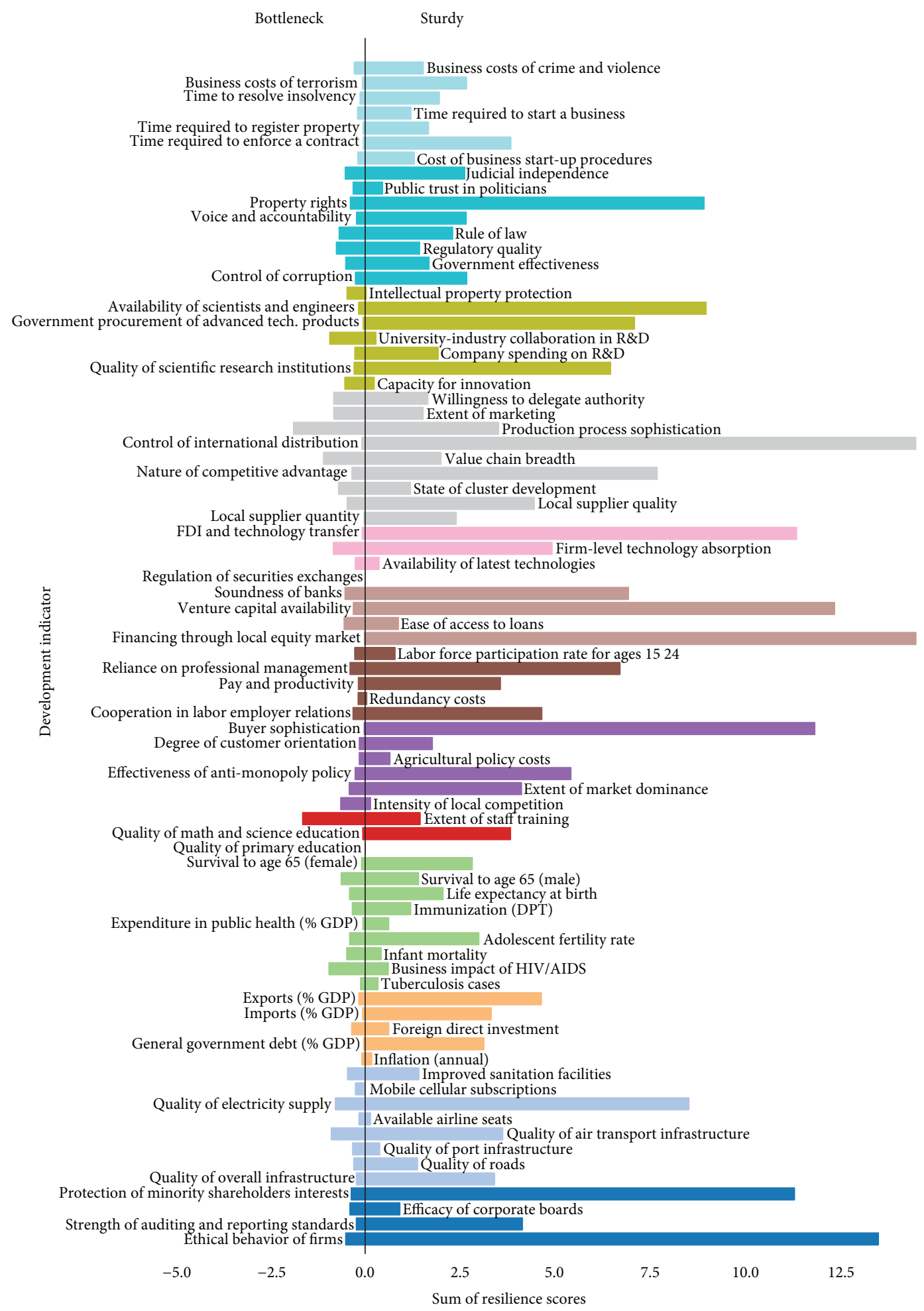

FIGURE 12: Sturdy policies and bottlenecks by development indicators. Bottlenecks: fragile policies in the $5^{\text {th }}$ percentile of relative priorities. Sturdy: resilient policies in the $95^{\text {th }}$ percentile of relative priorities.

Fifth, there is a set of sturdy policy issues that combine high levels of resilience with large budget allocations. In contrast, fragile policies with low budgetary priority are identified as the bottlenecks to development. Overall, there are more sturdy policies than bottlenecks. However, the latter should raise some flags in the development strategies of governments, since they represent major impairments to the economic development of their nations. 


\section{Appendix}

\section{Country-Level Analysis}

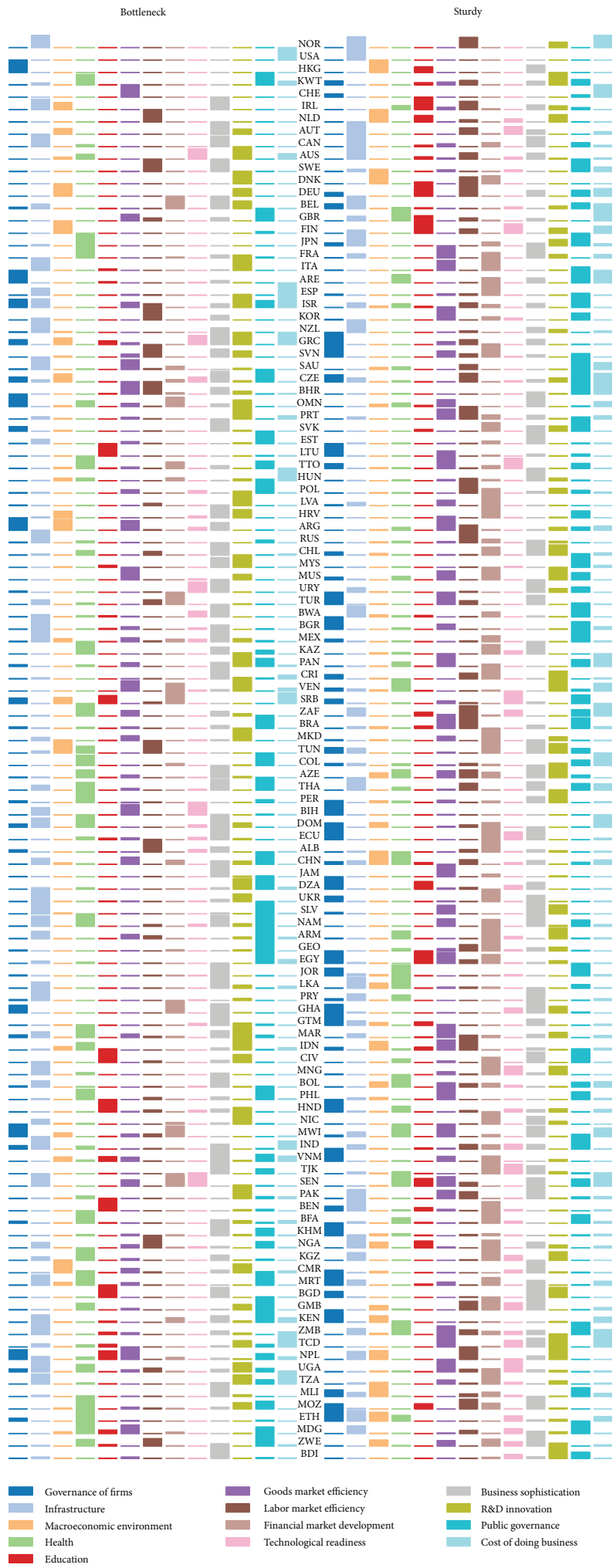

FIGURE 13: Sturdy policies and bottlenecks by development indicators by country (sorted by purchasing power parity income per capita). Bottlenecks: fragile policies in the $5^{\text {th }}$ percentile of relative priorities. Sturdy: resilient policies in the $95^{\text {th }}$ percentile of relative priorities. 


\section{Data Availability}

The data was obtained from public datasets from the World Bank and the World Economic Forum.

\section{Conflicts of Interest}

The authors declare that they have no conflicts of interest.

\section{References}

[1] D. Colander and R. Kupers, Complexity and the Art of Public Policy: Solving Society's Problems from the Bottom Up, Princeton University Press, 2014.

[2] L. Robbins, An Essay on the Nature and Significance of Economic Science, McMillan, London, UK, 2014.

[3] A. Zolli and A. Healy, Resilience: Why Things Bounce Back, Free Press, New York, NY, USA, 2012.

[4] H. Simon, "The architecture of complexity," Proceedings of the American Philosophical Society, vol. 106, no. 6, pp. 467-482, 1962.

[5] H. Simon, The Sciences of the Artificial, MIT Press, Cambridge, MA, USA, 1996.

[6] G. Castañeda, F. Chávez-Juárez, and O. A. Guerrero, "How do governments determine policy priorities? Studying development strategies through spillover networks," Journal of Economic Behavior \& Organization, vol. 154, pp. 335-361, 2018.

[7] G. Capano and J. J. Woo, "Resilience and robustness in policy design: a critical appraisal," Policy Sciences, vol. 50, no. 3, pp. 399-426, 2017.

[8] A. Barabási and M. Pósfai, Network Science, Cambridge University Press, Cambridge, UK, 2016.

[9] K. Anand, P. Gai, S. Kapadia, S. Brennan, and M. Willison, “A network model of financial system resilience," Journal of Economic Behavior \& Organization, vol. 85, pp. 219-235, 2013.

[10] D. Grigat and F. Caccioli, "Reverse stress testing interbank networks," Scientific Reports, vol. 7, no. 1, article 15616, 2017.

[11] K. Chmutina, G. Lizarralde, A. Dainty, and L. Bosher, "Unpacking resilience policy discourse," Cities, vol. 58, pp. 70-79, 2016.

[12] A. Duit, "Resilience thinking: lessons for public administration," Public Administration, vol. 94, no. 2, pp. 364-380, 2016.

[13] N. Galea, A. Powell, M. Loosemore, and L. Chappell, "Designing robust and revisable policies for gender equality: lessons from the Australian construction industry," Construction Management and Economics, vol. 33, no. 5-6, pp. 375-389, 2015.

[14] L. Ceriani and C. Gigliarano, "Multidimensional well-being: a Bayesian networks approach," Technical Report 399, ECINEQ, Society for the Study of Economic Inequality, 2016.

[15] E. N. Cinicioglu, G. Ulusoy, Ş. Önsel Ekici, F. Ülengin, and B. Ülengin, "Exploring the interaction between competitiveness of a country and innovation using Bayesian networks," Innovation and Development, vol. 7, no. 2, pp. 175-209, 2017.

[16] M. Czyżewska and T. Mroczek, "Bayesian approach to the process of identification of the determinants of innovativeness," e-Finanse: Financial Internet Quarterly, vol. 10, no. 2, pp. 44-56, 2014.

[17] P. Hoyer, A. Hyvärinen, R. Scheines et al., "Causal discovery of linear acyclic models with arbitrary distributions," in Proceedings of the Twenty-Fourth Conference on Uncertainty in Artificial Intelligence, UAI'08, pp. 282-289, Arlington, Virginia, USA, 2008.

[18] S. M. Smith, K. L. Miller, G. Salimi-Khorshidi et al., "Network modelling methods for FMRI," NeuroImage, vol. 54, no. 2, pp. 875-891, 2011.

[19] G. Massara, T. Di Matteo, and T. Aste, "Network filtering for big data: triangulated maximally filtered graph," Journal of Complex Networks, vol. 5, no. 2, pp. 161-178, 2017.

[20] A. Hyvärinen and S. Smith, "Pairwise likelihood ratios for estimation of non-Gaussian structural equation models," Journal of Machine Learning Research, vol. 14, pp. 111-152, 2013.

[21] R. Hausmann, B. Klinger, and R. Wagner, "Doing growth diagnostics in practice: a'mindbook'," Technical Report 177, Center for International Development at Harvard University, 2008.

[22] P. Rondo-Brovetto and I. Saliterer, "Comparing regions, cities, and communities: local government benchmarking as an instrument for improving performance and competitiveness," The Innovation Journal: The Public Sector Innovation Journal, vol. 12, no. 3, 2007.

[23] H. Werlin, "Bottlenecks to developments: studies from the World Bank's Economic Development Institute," Public Administration and Development, vol. 11, no. 3, pp. 189-191, 1991. 


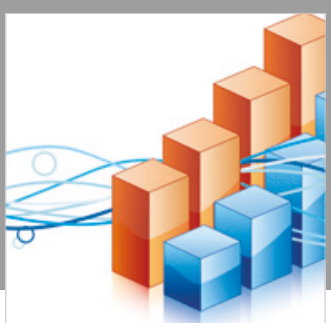

Advances in

Operations Research

\section{-n-m}
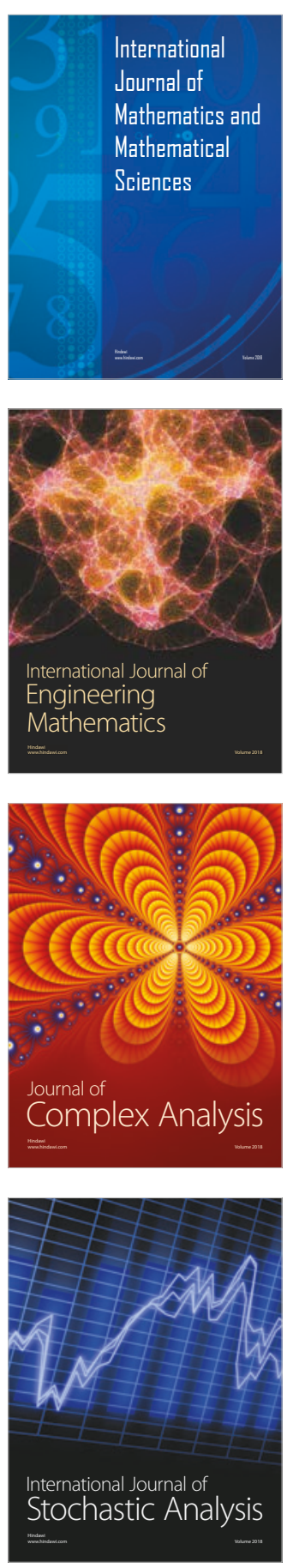
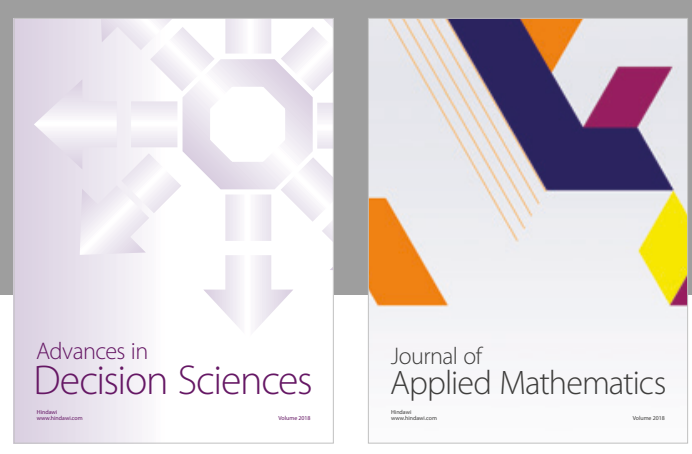

Journal of

Applied Mathematics
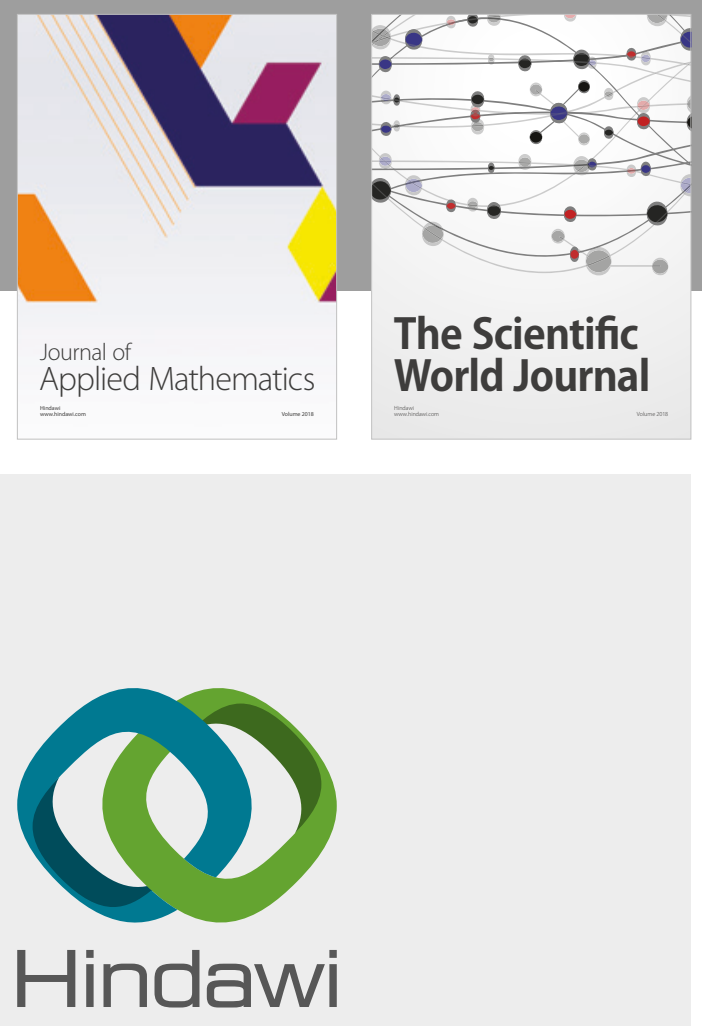

Submit your manuscripts at

www.hindawi.com

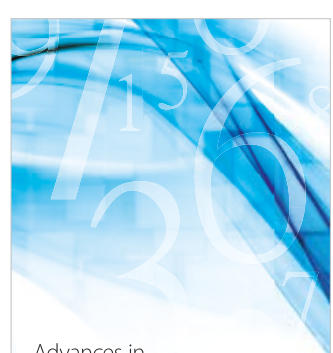

Advances in
Numerical Analysis
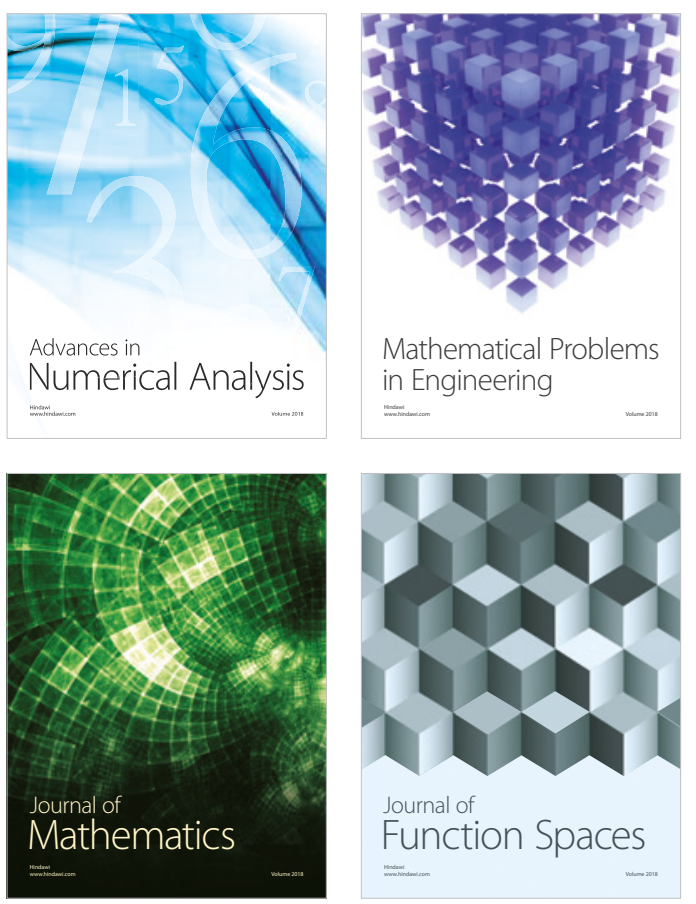

Mathematical Problems in Engineering

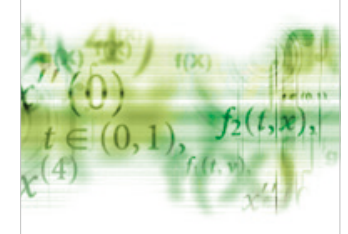

International Journal of

Differential Equations

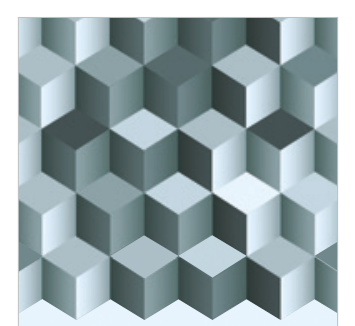

Journal of

Function Spaces
The Scientific

World Journal

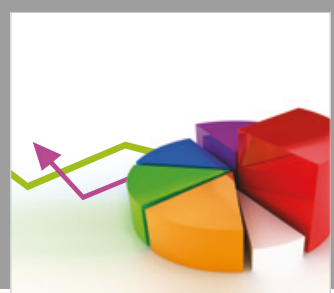

Journal of

Probability and Statistics
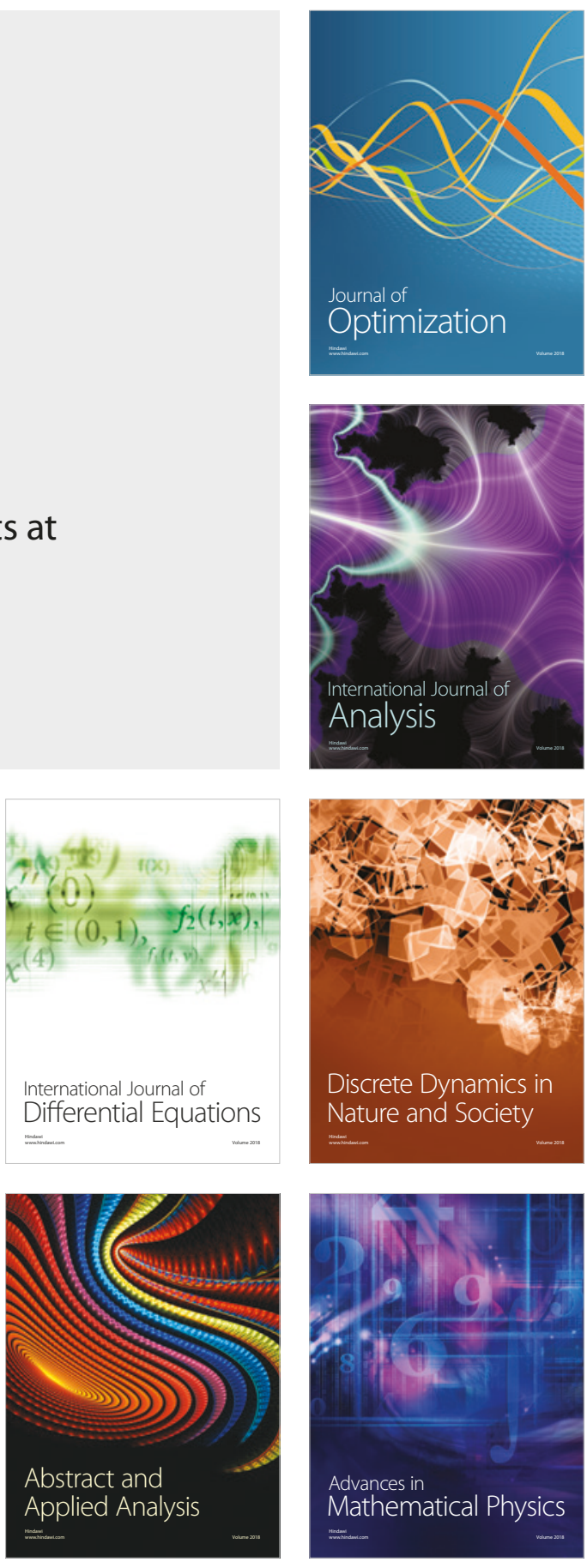